\title{
A Review of Regular Decompositions of Vector Fields: Continuous, Discrete, and Structure-Preserving
}

\author{
Ralf Hiptmair and Clemens Pechstein
}

\section{Introduction}

For a bounded Lipschitz domain $\Omega \subset \mathbb{R}^{3}$ recall the classical $L^{2}$-orthogonal Helmholtz decompositions

$$
\mathbf{L}^{2}(\Omega)=\nabla H_{0}^{1}(\Omega) \oplus \mathbf{H}(\operatorname{div} 0, \Omega)=\nabla H^{1}(\Omega) \oplus \mathbf{H}_{0}(\operatorname{div} 0, \Omega),
$$

see, e.g., [9, Ch. XI, Sect. I]. They can be used to derive decompositions of (subspaces of) $\mathbf{H}($ curl, $\Omega$ ):

$$
\begin{aligned}
\mathbf{H}_{0}(\operatorname{curl}, \Omega) & =\nabla H_{0}^{1}(\Omega) \oplus \mathbf{X}_{N}(\Omega), & \mathbf{X}_{N}(\Omega):=\mathbf{H}_{0}(\operatorname{curl}, \Omega) \cap \mathbf{H}(\operatorname{div} 0, \Omega), \\
\mathbf{H}(\operatorname{curl}, \Omega) & =\nabla H^{1}(\Omega) \oplus \mathbf{X}_{T}(\Omega), & \mathbf{X}_{T}(\Omega):=\mathbf{H}(\operatorname{curl}, \Omega) \cap \mathbf{H}_{0}(\operatorname{div} 0, \Omega) .
\end{aligned}
$$

If the domain $\Omega$ is convex then the respective complementary space, $\mathbf{X}_{N}(\Omega)$ or $\mathbf{X}_{T}(\Omega)$, is continuously embedded in the space $\mathbf{H}^{1}(\Omega)$ of vector fields with Cartesian components in $H^{1}(\Omega)$, cf. [1]. Then one can, for instance, write any $\mathbf{u} \in \mathbf{H}(\mathbf{c u r l}, \Omega)$ as

$$
\mathbf{u}=\nabla p+\mathbf{z}
$$

\section{R. Hiptmair $(\varangle)$}

Seminar for Applied Mathematics, ETH Zürich, Zürich, Switzerland

e-mail: hiptmair@sam.math.ethz.ch

C. Pechstein

Dassault Systèmes, Darmstadt, Germany

e-mail: clemens.pechstein@3ds.com 
with $p \in H^{1}(\Omega)$ and $\mathbf{z} \in \mathbf{H}^{1}(\Omega)$. Since $\|\nabla p\|_{\mathbf{L}^{2}(\Omega)} \leq\|\mathbf{u}\|_{\mathbf{L}^{2}(\Omega)}$ one obtains (using the continuous embedding) the stability property ${ }^{1}$

$$
\|\nabla p\|_{\mathbf{L}^{2}(\Omega)}+\|\mathbf{z}\|_{\mathbf{H}^{1}(\Omega)} \leq C\|\mathbf{z}\|_{\mathbf{H}(\operatorname{curl}, \Omega)} .
$$

A similar decomposition can be found for $\mathbf{u} \in \mathbf{H}_{0}(\mathbf{c u r l}, \Omega)$.

Generally, a decomposition of form (1) with the stability property (2) is called regular decomposition, even if $\mathbf{L}^{2}$-orthogonality does not hold. Actually, it turns out that (1)-(2) can be achieved even in cases where $\Omega$ is non-convex, in particular on non-smooth domains, or in cases where $\Omega$ or its boundary have non-trivial topology; only the $\mathbf{L}^{2}$-orthogonality has to be sacrificed, cf. [20].

Noting that $\nabla H^{1}(\Omega)$ is contained in the kernel of the curl operator and thatunder mild smoothness assumptions on the domain - the whole kernel is spanned by $\nabla H^{1}(\Omega)$ plus a finite-dimensional co-homology space [15, Sect. 4] one can achieve a second decomposition,

$$
\mathbf{u}=\mathbf{h}+\mathbf{z}
$$

with $\mathbf{h} \in \operatorname{ker}\left(\operatorname{curl}_{\mid \mathbf{H}(\operatorname{curl}, \Omega)}\right)$ and $\mathbf{z} \in \mathbf{H}^{1}(\Omega)$, where

$$
\|\mathbf{h}\|_{\mathbf{L}^{2}(\Omega)} \leq C\|\mathbf{u}\|_{\mathbf{L}^{2}(\Omega)}, \quad\|\mathbf{z}\|_{\mathbf{H}^{1}(\Omega)} \leq C\|\operatorname{curl} \mathbf{u}\|_{\mathbf{L}^{2}(\Omega)} .
$$

The second stability estimate states that if $\mathbf{u}$ is already in the kernel of the curl operator, then $\mathbf{z}$ is zero. Hence, (1) the operator mapping $\mathbf{u}$ to $\mathbf{h}$ is a projection onto the kernel space and (2) the complement operator projects $\mathbf{u}$ to the function $\mathbf{z}$ of higher regularity $\mathbf{H}^{1}(\Omega)$. For trivial topology of $\Omega$ and $\partial \Omega$, the two decompositions (1)(2) and (3)-(4) coincide.

As a few among many more [17, Sect. 1.5], we would like to highlight two important applications of these regular decompositions.

1. The second form (3)-(4), in the sequel called rotation-bounded decomposition, can be used to show that the operator underlying a certain boundary value problem for Maxwell's equations is a Fredholm operator. The key point is that the complement space of the kernel (from the view of the mentioned projections) is $\mathbf{H}^{1}(\Omega)$ which is compactly embedded in $\mathbf{L}^{2}(\Omega)$, see e.g., $[14,16]$ and references therein.

2. The first form (1)-(2), in the sequel called gradient-based decomposition, has been used to generate stable three-term splittings of a finite element subspace of $\mathbf{H}(\mathbf{c u r l}, \Omega)$, cf. [19-21, 23], which allows the construction of so-called fictitious or auxiliary space preconditioners for the ill-conditioned system matrix underlying the discretized Maxwell equations.

\footnotetext{
${ }^{1}$ Here and below $C$ stands for a positive "generic constant" that may depend only on $\Omega$, unless specified otherwise.
} 
In both applications, it is desirable to obtain the decompositions for minimal smoothness of the domain, e.g., Lipschitz domains, which are not necessarily convex. Moreover, it is also desirable to go beyond decompositions of the entire space $\mathbf{H}(\mathbf{c u r l}, \Omega)$ and extend them to subspaces for which the appropriate trace vanishes on a "Dirichlet part" $\Gamma_{D}$ of the boundary. In this case traces of the two summands should also vanish on $\Gamma_{D}$.

In the present paper, we provide regular decompositions of both types for subspaces of $\mathbf{H}($ curl, $\Omega$ ) (in Sect. 3) and $\mathbf{H}(\operatorname{div}, \Omega$ ) (in Sect. 4) comprising functions with vanishing trace on a part $\Gamma_{D}$ of the boundary $\partial \Omega$ for Lipschitz domains $\Omega$ of arbitrary topology. In particular, $\Omega$ is allowed to have handles, and $\partial \Omega$ and $\Gamma_{D}$ may have several connected components. The Dirichlet boundary $\Gamma_{D}$ must satisfy a certain smoothness assumption that we shall introduce in Sect. 2. In addition to the stability estimates (2) and (4), we show that the decompositions are stable even in $\mathbf{L}^{2}(\Omega)$.

In the final part of the manuscript, in Sect. 5, we establish regular decompositions of spaces of Whitney forms, which are lowest-order conforming finite element subspaces of $\mathbf{H}(\mathbf{c u r l}, \Omega)$ and $\mathbf{H}(\operatorname{div}, \Omega)$, respectively, built upon simplicial triangulations of $\Omega$.

This note is based on [17] and is an abridged version of [18]. Please refer to this latter preprint for complete proofs of the results quoted below.

\section{Preliminaries}

Since subtle geometric arguments will play a major role for parts of the theory, we start with a precise characterization of the geometric setting: Let $\Omega \subset \mathbb{R}^{3}$ be an open, bounded, connected Lipschitz domain. ${ }^{2}$ We write $\mathrm{d}(\Omega)$ for its diameter. Its boundary $\Gamma:=\partial \Omega$ is partitioned according to $\Gamma=\Gamma_{D} \cup \Sigma \cup \Gamma_{N}$, with relatively open sets $\Gamma_{D}$ and $\Gamma_{N}$. We assume that this provides a piecewise $C^{1}$ dissection of $\partial \Omega$ in the sense of [12, Definition 2.2]. Sloppily speaking, this means that $\Sigma$ is the union of closed curves that are piecewise $C^{1}$.

Under the above assumptions on $\Omega$ and $\Gamma_{D}$, [12, Lemma 4.4] guarantees the existence of an open Lipschitz neighborhood $\Omega_{\Gamma}$ ("Lipschitz collar") of $\Gamma$ and of a "bulge" $\Upsilon_{D} \subset \Omega_{\Gamma} \backslash \Omega$. We recall the properties of bulge domains from [12, Sect. 2, Thm. 2.3], also stated in [17, Thm. 2.2]:

Theorem 1 (Bulge-Augmented Domain) There exists a Lipschitz domain $\Upsilon_{D} \subset$ $\mathbb{R}^{3} \backslash \bar{\Omega}$, such that $\bar{\Upsilon}_{D} \cap \bar{\Omega}=\Gamma_{D}, \Omega^{e}:=\Upsilon_{D} \cup \Gamma_{D} \cup \Omega$ is Lipschitz, $\mathrm{d}\left(\Omega^{e}\right) \leq 2 \mathrm{~d}(\Omega)$, and $\bar{\Upsilon}_{D} \subset \Omega_{\Gamma}$. Moreover, each connected component $\Gamma_{D, k}$ of $\Gamma_{D}$ corresponds to a connected component $\Upsilon_{D, k}$ of $\Upsilon_{D}$, and these have positive distance from each other.

\footnotetext{
${ }^{2}$ Strongly Lipschitz, in the sense that the boundary is locally the graph of a Lipschitz continuous function.
} 
Let

$$
\begin{aligned}
H_{\Gamma_{D}}^{1}(\Omega) & :=\left\{u \in H^{1}(\Omega):(\gamma u)_{\mid \Gamma_{D}}=0\right\}, \\
\mathbf{H}_{\Gamma_{D}}(\operatorname{curl}, \Omega) & :=\left\{\mathbf{u} \in \mathbf{H}(\mathbf{c u r l}, \Omega):\left(\gamma_{\tau} \mathbf{u}\right)_{\mid \Gamma_{D}}=0\right\}, \\
\mathbf{H}_{\Gamma_{D}}(\operatorname{div}, \Omega) & :=\left\{\mathbf{u} \in \mathbf{H}(\operatorname{div}, \Omega):\left(\gamma_{n} \mathbf{u}\right)_{\mid \Gamma_{D}}=0\right\},
\end{aligned}
$$

denote the standard Sobolev spaces where the distributional gradient, curl, or divergence is in $L^{2}$ and where the pointwise trace $\gamma u$, the tangential trace $\gamma_{\tau} \mathbf{u}$, or the normal trace $\gamma_{n} \mathbf{u}$, respectively, vanishes on the Dirichlet boundary $\Gamma_{D}$, see e.g. $[3,6,26]$. These space are linked via the de Rham complex,

$$
\mathcal{K}_{\Gamma_{D}}(\Omega) \stackrel{\mathrm{id}}{\longrightarrow} H_{\Gamma_{D}}^{1}(\Omega) \stackrel{\nabla}{\longrightarrow} \mathbf{H}_{\Gamma_{D}}(\operatorname{curl}, \Omega) \stackrel{\text { curl }}{\longrightarrow} \mathbf{H}_{\Gamma_{D}}(\operatorname{div}, \Omega) \stackrel{\operatorname{div}}{\longrightarrow} L^{2}(\Omega),
$$

where

$$
\mathcal{K}_{\Gamma_{D}}(\Omega):=\left\{v \in H_{\Gamma_{D}}^{1}(\Omega): v=\mathrm{const}\right\}= \begin{cases}\operatorname{span}\{1\}, & \text { if } \Gamma_{D}=\emptyset \\ \{0\}, & \text { otherwise }\end{cases}
$$

The range of each operator in (5) lies in the kernel space of the succeeding one, cf. [3, Lemma 2.2]. We define

$$
\begin{aligned}
\mathbf{H}_{\Gamma_{D}}(\operatorname{curl} 0, \Omega) & :=\left\{\mathbf{v} \in \mathbf{H}_{\Gamma_{D}}(\operatorname{curl}, \Omega): \operatorname{curl} \mathbf{v}=0\right\}, \\
\mathbf{H}_{\Gamma_{D}}(\operatorname{div} 0, \Omega) & :=\left\{\mathbf{v} \in \mathbf{H}_{\Gamma_{D}}(\operatorname{div}, \Omega): \operatorname{div} \mathbf{v}=0\right\} .
\end{aligned}
$$

Barring topological obstructions these kernels can be represented through potentials: Let $\beta_{1}(\Omega)$ denote the first Betti number of $\Omega$ (the number of "handles") and $\beta_{2}(\Omega)$ the second Betti number (the number of connected components of $\partial \Omega$ minus one). By the very definition of the Betti numbers as dimensions of co-homology spaces we have

$$
\begin{aligned}
& \beta_{1}(\Omega)=0 \quad \Longrightarrow \quad \mathbf{H}(\operatorname{curl} 0, \Omega)=\nabla H^{1}(\Omega), \\
& \beta_{2}(\Omega)=0 \quad \Longrightarrow \quad \mathbf{H}(\operatorname{div} 0, \Omega)=\operatorname{curl} \mathbf{H}(\operatorname{curl}, \Omega),
\end{aligned}
$$

cf. [26]. We call $\Omega$ topologically trivial if $\beta_{1}(\Omega)=\beta_{2}(\Omega)=0$.

\section{Regular Decompositions and Potentials Related to H(curl)}

Throughout we rely on the properties of $\Omega$ and $\Gamma_{D}$ as introduced in Sect. 2 and use the notations from Theorem 1 . We write $C$ for positive "generic constants" and 
say that a constant "depends only on the shape of $\Omega$ and $\Gamma_{D}$ ", if it depends on the geometric setting alone, but is invariant with respect to similarity transformations. To achieve this the diameter of $\Omega$ will have to enter the estimates; we denote it by $\mathrm{d}(\Omega)$.

\subsection{Gradient-Based Regular Decomposition of $\mathrm{H}(\mathrm{curl})$}

The following theorem is essentially [17, Thm. 2.1].

Theorem 2 (Gradient-Based Regular Decomposition of $\mathbf{H}\left(\right.$ curl)) Let $\left(\Omega, \Gamma_{D}\right)$ satisfy the assumptions of Sect. 2. Then for each $\mathbf{u} \in \mathbf{H}_{\Gamma_{D}}(\mathbf{c u r l}, \Omega)$ there exist $\mathbf{z} \in$ $\mathbf{H}_{\Gamma_{D}}^{1}(\Omega)$ and $p \in H_{\Gamma_{D}}^{1}(\Omega)$ depending linearly on $\mathbf{u}$ such that

$$
\begin{aligned}
\mathbf{u} & =\mathbf{z}+\nabla p, \\
\|\mathbf{z}\|_{0, \Omega}+\|\nabla p\|_{0, \Omega} & \leq C\|\mathbf{u}\|_{0, \Omega}, \\
\|\nabla \mathbf{z}\|_{0, \Omega}+\frac{1}{\mathrm{~d}(\Omega)}\|\mathbf{z}\|_{0, \Omega} & \leq C\|\operatorname{curl} \mathbf{u}\|_{0, \Omega}+\frac{1}{\mathrm{~d}(\Omega)}\|\mathbf{u}\|_{0, \Omega},
\end{aligned}
$$

with constants depending only on the shape of $\Omega$ and $\Gamma_{D}$, but not on $\mathrm{d}(\Omega)$.

Remark 1 An early decomposition of a subspace of $\mathbf{H}(\mathbf{c u r l}, \Omega) \cap \mathbf{H}(\operatorname{div}, \Omega)$ into a regular part in $\mathbf{H}^{1}(\Omega)$ and a singular part in $\nabla H^{1}(\Omega)$ can be found in [4] and in [5, Proposition 5.1], see also [7, Sect. 3] and references therein. Theorem 2 was proved in [14, Lemma 2.4] for the case of $\Gamma_{D}=\partial \Omega$ and without the $\mathbf{L}^{2}$ stability estimate, following [5, Proposition 5.1]. Pasciak and Zhao [28, Lemma 2.2] provided a version for simply connected $\Omega$ and the case $\Gamma_{D}=\partial \Omega$ with pure $\mathbf{L}^{2}$-stability, but $p$ is only constant on each connected component of $\partial \Omega$ (see also Theorem 5 and Remark 3). This result was refined in [24, Thm. 3.1]. For the case $\Gamma_{D}=\varnothing$, [14, Lemma 2.4] gives a similar decomposition but $\nabla p$ must be replaced by an element from $\mathbf{H}(\mathbf{c u r l} 0, \Omega)$ in general. Finally, Theorem 2 without the pure $\mathbf{L}^{2}$-stability was proved in [20, Thm. 5.2]. ${ }^{3}$

Remark 2 The constant $C$ in Theorem 2 depends mainly on the stability constants of key extension operators. If the bulge $\Upsilon_{D}$ has multiple components $\Upsilon_{D, k}$, the final estimate will depend on the relative distances between $\Upsilon_{D, k}, \Upsilon_{D, \ell}, k \neq \ell$ and the ratios $\mathrm{d}\left(\Upsilon_{D, k}\right) / \mathrm{d}(\Omega)$.

\footnotetext{
${ }^{3}$ This reference contains a typo which is easily identified when inspecting the proof: In general, $\mathbf{z}$ cannot be estimated in terms of $\|$ curl $\mathbf{u} \|_{0, \Omega}$ but one must use the full $\mathbf{H}$ (curl) norm.
} 
Remark 3 If $\Gamma_{D}=\partial \Omega$, one obtains only $p \in H^{1}(\Omega)$ being constant on each connected component of $\Gamma_{D}$ but the improved bound

$$
\|\nabla \mathbf{z}\|_{0, \Omega}+\mathrm{d}(\Omega)^{-1}\|\mathbf{z}\|_{0, \Omega} \leq C\|\operatorname{curl} \mathbf{u}\|_{0, \Omega} .
$$

Results on regular decompositions in this special case can be found in [24, 28].

\subsection{Regular Potentials for Some Divergence-Free Functions}

Let the domain $\Omega$ and the Dirichlet boundary part $\Gamma_{D}$ be as introduced in Sect. 2 and let $\Gamma_{i}, i=0, \ldots, \beta_{2}(\Omega)$, denote the connected components of $\partial \Omega$, where $\beta_{2}(\Omega)$ is the second Betti number of $\Omega$.

We define the space 4

$$
\mathbf{H}_{\Gamma_{D}}(\operatorname{div} 00, \Omega):=\left\{\mathbf{q} \in \mathbf{H}_{\Gamma_{D}}(\operatorname{div} 0, \Omega):\left\langle\gamma_{n} \mathbf{q}, 1\right\rangle_{\Gamma_{i}}=0, i=0, \ldots, \beta_{2}(\Omega)\right\}
$$

Above $\gamma_{n}$ denotes the normal trace operator, and the duality pairing is that between $H^{-1 / 2}\left(\Gamma_{i}\right)$ and $H^{1 / 2}\left(\Gamma_{i}\right)$. If $\Gamma_{D}=\emptyset$ we simply drop the subscript $\Gamma_{D}$. Obviously,

$$
\mathbf{H}_{\Gamma_{D}}(\operatorname{div} 00, \Omega) \subset \mathbf{H}(\operatorname{div} 00, \Omega) .
$$

The next result identifies the above space as the range of the curl operator.

Theorem 3 (Regular Potential of Range(curl)) Let $\left(\Omega, \Gamma_{D}\right)$ be as in Sect. 2 and assume in addition that each connected component $\Upsilon_{D, k}$ of the bulge has vanishing first Betti number, $\beta_{1}\left(\Upsilon_{D, k}\right)=0$. Then

$$
\mathbf{H}_{\Gamma_{D}}(\operatorname{div} 00, \Omega)=\operatorname{curl} \mathbf{H}_{\Gamma_{D}}(\operatorname{curl}, \Omega)=\operatorname{curl} \mathbf{H}_{\Gamma_{D}}^{1}(\Omega),
$$

and for each $\mathbf{q} \in \mathbf{H}_{\Gamma_{D}}(\operatorname{div} 00, \Omega)$ there exists $\boldsymbol{\psi} \in \mathbf{H}_{\Gamma_{D}}^{1}(\Omega)$ depending linearly on q such that

$$
\operatorname{curl} \boldsymbol{\psi}=\mathbf{q} \text { and }\|\nabla \boldsymbol{\psi}\|_{0, \Omega}+\frac{1}{\mathrm{~d}(\Omega)}\|\boldsymbol{\psi}\|_{0, \Omega} \leq C\|\mathbf{q}\|_{0, \Omega},
$$

where $C$ depends only on the shape of $\Omega$ and $\Gamma_{D}$, but not on $\mathrm{d}(\Omega)$

\footnotetext{
${ }^{4}$ Alternatively we can define $\mathbf{H}_{\Gamma_{D}}(\operatorname{div} 00, \Omega)$ as the functions in $\mathbf{H}_{\Gamma_{D}}(\operatorname{div} 0, \Omega)$ orthogonal to the harmonic Dirichlet fields $\mathbf{H}(\operatorname{div} 0, \Omega) \cap \mathbf{H}_{0}(\operatorname{curl} 0, \Omega)$.
} 
Remark 4 For the case that $\Gamma_{D}=\emptyset$, we reproduce the classical result

$$
\mathbf{H}(\operatorname{div} 00, \Omega)=\operatorname{curl} \mathbf{H}(\operatorname{curl}, \Omega)=\operatorname{curl} \mathbf{H}^{1}(\Omega),
$$

see [11, Thm. 3.4]. In that case, Step 4 of the proof can be left out and $\psi=\mathbf{w}_{1}$ which is why $\operatorname{div} \psi=0$ in $\Omega$. This property, however, is lost in the general case.

\subsection{Rotation-Bounded Regular Decomposition of $\mathbf{H}(\mathrm{curl})$}

We can now formulate another new variety of regular decompositions, for which the $\mathbf{H}^{1}$-component will vanish for curl-free fields.

Theorem 4 (Rotation-Bounded Regular Decomposition of $\mathbf{H}$ (curl) (I)) Let $\left(\Omega, \Gamma_{D}\right)$ be as in Sect. 2 and assume, in addition, that each connected component $\Upsilon_{D, k}$ of the bulge has vanishing first Betti number, $\beta_{1}\left(\Upsilon_{D, k}\right)=0$. Then, for each $\mathbf{u} \in \mathbf{H}_{\Gamma_{D}}$ (curl, $\Omega$ ) there exist $\mathbf{z} \in \mathbf{H}_{\Gamma_{D}}^{1}(\Omega)$ and a curl-free vector field $\mathbf{h} \in \mathbf{H}_{\Gamma_{D}}(\mathbf{c u r l} 0, \Omega)$, depending linearly on $\mathbf{u}$ such that

$$
\begin{aligned}
\mathbf{u} & =\mathbf{z}+\mathbf{h}, \\
\|\mathbf{h}\|_{0, \Omega} & \leq\|\mathbf{u}\|_{0, \Omega}+C \mathrm{~d}(\Omega) \| \text { curl } \mathbf{u} \|_{0, \Omega}, \\
\|\nabla \mathbf{z}\|_{0, \Omega}+\frac{1}{\mathrm{~d}(\Omega)}\|\mathbf{z}\|_{0, \Omega} & \leq C\|\operatorname{curl} \mathbf{u}\|_{0, \Omega},
\end{aligned}
$$

where $C$ depends only on the shape of $\Omega$ and $\Gamma_{D}$, but not on $\mathrm{d}(\Omega)$.

Remark 5 The constant $C$ in Theorem 4 depends essentially on the stability constants of the divergence-free extension operator $E_{\Omega^{e}}^{\text {div, } 0}$ and the (adapted) Stein extension operator $E_{\Upsilon_{D}}^{\nabla, \text { Stein }}$.

Another stronger version of the rotation-bounded regular decomposition of $\mathbf{H}(\mathbf{c u r l})$ gets rid of the assumptions on the topology of the Dirichlet boundary and has improved stability properties (though with less explicit constants).

Theorem 5 (Rotation-Bounded Regular Decomposition of $\mathbf{H}$ (curl) (II)) Let $\left(\Omega, \Gamma_{D}\right)$ be as in Sect. 2. Then for each $\mathbf{u} \in \mathbf{H}_{\Gamma_{D}}(\mathbf{c u r l}, \Omega)$ there exist $\mathbf{z} \in \mathbf{H}_{\Gamma_{D}}^{1}(\Omega)$ and a curl-free $\mathbf{h} \in \mathbf{H}_{\Gamma_{D}}$ (curl $\left.0, \Omega\right)$ depending linearly on $\mathbf{u}$ such that

$$
\begin{aligned}
\mathbf{u} & =\mathbf{z}+\mathbf{h}, \\
\|\mathbf{z}\|_{0, \Omega}+\|\mathbf{h}\|_{0, \Omega} & \leq C\|\mathbf{u}\|_{0, \Omega}, \\
\|\nabla \mathbf{z}\|_{0, \Omega}+\mathrm{d}(\Omega)^{-1}\|\mathbf{z}\|_{0, \Omega} & \leq C \| \text { curl } \mathbf{u} \|_{0, \Omega},
\end{aligned}
$$

where $C$ depends only on the shape of $\Omega$ and $\Gamma_{D}$, but not on $\mathrm{d}(\Omega)$. 
Remark 6 For the case $\Gamma_{D}=\partial \Omega$ the result of the theorem is already proved by Remark 3 since we obtain $\mathbf{u}=\mathbf{z}+\nabla p$ with $\nabla p \in \nabla H_{0 \text {,const }}^{1}(\Omega)=\mathbf{H}_{0}($ curl, $\Omega)$.

Remark 7 We would like to emphasize that both in Theorems 2 and 5, the domain $\Omega$ may be non-convex, non-smooth, and may have non-trivial topology: It may have handles and its boundary may have multiple components. Also the Dirichlet boundary $\Gamma_{D}$ may have multiple components, each of which with non-trivial topology. Moreover, we have the pure $\mathbf{L}^{2}(\Omega)$-stability in both theorems. In this sense, the results of Theorems 2 and 5 are superior to those found, e.g., in [7, Thm 3.4], [19] or the more recent ones in [8, Thm. 2.3], [22].

Remark 8 If $\Omega$ has vanishing first Betti number, $\beta_{1}(\Omega)=0$, then $\mathbf{H}_{\Gamma_{D}}(\mathbf{c u r l} 0, \Omega)$ $=\nabla H_{\Gamma_{D} \text {, const }}^{1}(\Omega)$. Hence, we can split each $\mathbf{u} \in \mathbf{H}_{\Gamma_{D}}(\mathbf{c u r l}, \Omega)$ into $\mathbf{z} \in \mathbf{H}_{\Gamma_{D}}^{1}(\Omega)$ and $\nabla p$ with $p \in H^{1}(\Omega)$ being constant on each connected component of $\Gamma_{D}$. If $\Gamma_{D}$ is connected, then $p \in H_{\Gamma_{D}}^{1}(\Omega)$. Summarizing, if $\Omega$ has no handles and if $\Gamma_{D}$ is connected, then we have the combined features of Theorems 2 and 5.

Finally, we mention that the regular decomposition theorems spawn projection operators that play a fundamental role in the analysis of weak formulations of Maxwell's equations in frequency domain [14, Sect. 5].

Corollary 1 Let $\left(\Omega, \Gamma_{D}\right)$ be as in Sect.2. Then there exist continuous projection operators $\mathrm{R}: \mathbf{H}_{\Gamma_{D}}(\operatorname{curl}, \Omega) \rightarrow \mathbf{H}_{\Gamma_{D}}^{1}(\Omega)$ and $\mathrm{N}: \mathbf{H}_{\Gamma_{D}}(\mathbf{c u r l}, \Omega) \rightarrow$ $\mathbf{H}_{\Gamma_{D}}(\operatorname{curl} 0, \Omega)$ such that $\mathrm{R}+\mathrm{N}=$ id and

$$
\|\mathrm{R} \mathbf{v}\|_{\mathbf{H}^{1}(\Omega)}+\|\mathrm{Nv}\|_{\mathbf{L}^{2}(\Omega)} \leq C\|\mathbf{v}\|_{\mathbf{H}(\mathbf{c u r l}, \Omega)} \quad \forall \mathbf{v} \in \mathbf{H}(\text { curl }, \Omega),
$$

where $C$ is a constant independent of $\mathbf{v}$. Moreover, F: $\mathbf{H}_{\Gamma_{D}}(\mathbf{c u r l}, \Omega) \rightarrow$ $\mathbf{H}_{\Gamma_{D}}(\mathbf{c u r l}, \Omega)$ defined by $\mathbf{F v}:=\mathbf{R v}-\mathrm{Nv}$ is an isomorphism.

Remark 9 The $\mathbf{L}^{2}$-estimates from Theorem 4 then show that the corresponding operator $\mathrm{R}$ can be extended to a continuous operator mapping from $\mathbf{L}^{2}(\Omega)$ to $\mathbf{L}^{2}(\Omega)$.

\section{Regular Decompositions and Potentials Related to H(div)}

The developments of this section are largely parallel to those of Sect. 3 with some new aspects concerning extensions and topological considerations.

\subsection{Rotation-Based Regular Decomposition of H(div)}

The following theorem is the $\mathbf{H}$ (div)-counterpart of Theorem 2 . 
Theorem 6 (Rotation-Based Regular Decomposition of $\mathbf{H}(\mathrm{div}))$ Let $\left(\Omega, \Gamma_{D}\right)$ satisfy the assumptions made in Sect. 2. Then for each $\mathbf{v} \in \mathbf{H}_{\Gamma_{D}}(\operatorname{div}, \Omega)$ there exist $\mathbf{z} \in \mathbf{H}_{\Gamma_{D}}^{1}(\Omega)$ and $\mathbf{q} \in \mathbf{H}_{\Gamma_{D}}^{1}(\Omega)$ depending linearly on $\mathbf{v}$ such that

$$
\begin{aligned}
\mathbf{v} & =\mathbf{z}+\operatorname{curl} \mathbf{q}, \\
\|\mathbf{z}\|_{0, \Omega}+\|\operatorname{curl} \mathbf{q}\|_{0, \Omega}+\frac{1}{\mathrm{~d}(\Omega)}\|\mathbf{q}\|_{0, \Omega} & \leq C\|\mathbf{v}\|_{0, \Omega}, \\
\|\nabla \mathbf{z}\|_{0, \Omega}+\frac{1}{\mathrm{~d}(\Omega)}\|\mathbf{z}\|_{0, \Omega}+\frac{1}{\mathrm{~d}(\Omega)}\|\nabla \mathbf{q}\|_{0, \Omega} & \leq C\left(\|\operatorname{curl} \mathbf{v}\|_{0, \Omega}+\frac{1}{\mathrm{~d}(\Omega)}\|\mathbf{v}\|_{0, \Omega}\right),
\end{aligned}
$$

with constant $C$ depending only on the shape of $\Omega$ and $\Gamma_{D}$, but not on $\mathrm{d}(\Omega)$.

\subsection{Regular Potential with Prescribed Divergence}

The next result carries Theorem 3 over to $\mathbf{H}$ (div).

Theorem 7 (Regular Potentials for the Image Space of div) Let $\left(\Omega, \Gamma_{D}\right)$ be as in Sect. 2 and, in addition, assume that each connected component $\Upsilon_{D, k}$ of the bulge has a connected boundary, i.e., $\beta_{2}\left(\Upsilon_{D, k}\right)=0$. Then

$$
L^{2}(\Omega)=\operatorname{div} \mathbf{H}_{\Gamma_{D}}(\operatorname{div}, \Omega)=\operatorname{div} \mathbf{H}_{\Gamma_{D}}^{1}(\Omega) .
$$

Moreover, for each $v \in L^{2}(\Omega)$ there exists $\mathbf{q} \in \mathbf{H}_{\Gamma_{D}}^{1}(\Omega)$ depending linearly on $v$ such that, with a constant $C$ depending on $\Omega$ and $\Gamma_{D}$ but not on $\mathrm{d}(\Omega)$,

$$
\operatorname{div} \mathbf{q}=v \quad \text { and } \quad\|\nabla \mathbf{q}\|_{0, \Omega}+\frac{1}{\mathrm{~d}(\Omega)}\|\mathbf{q}\|_{0, \Omega} \leq C\|v\|_{0, \Omega} .
$$

\subsection{Divergence-Bounded Regular Decompositions of $\mathbf{H}(\mathrm{div})$}

We can now formulate other variants of regular decompositions of $\mathbf{H}$ (div) in analogy to what we did in Sect. 3.3.

Theorem 8 (Divergence-Bounded Regular Decomposition of $\mathbf{H}$ (div) (I)) Let $\left(\Omega, \Gamma_{D}\right)$ be as in Sect.2. In addition, assume that each connected component $\Upsilon_{D, k}$ of the bulge has a connected boundary, i.e., $\beta_{2}\left(\Upsilon_{D, k}\right)=0$. Then, for each $\mathbf{v} \in \mathbf{H}_{\Gamma_{D}}(\operatorname{div}, \Omega)$ there exists $\mathbf{z} \in \mathbf{H}_{\Gamma_{D}}^{1}(\Omega)$ and a divergence-free vector field 
$\mathbf{h} \in \mathbf{H}_{\Gamma_{D}}(\operatorname{div} 0, \Omega)$ depending linearly on $\mathbf{v}$ such that

$$
\begin{aligned}
\mathbf{v} & =\mathbf{z}+\mathbf{h}, \\
\|\mathbf{h}\|_{0, \Omega} & \leq\|\mathbf{v}\|_{0, \Omega}+C \mathrm{~d}(\Omega)\|\operatorname{div} \mathbf{v}\|_{0, \Omega}, \\
\|\nabla \mathbf{z}\|_{0, \Omega}+\frac{1}{\mathrm{~d}(\Omega)}\|\mathbf{z}\|_{0, \Omega} & \leq C\|\operatorname{div} \mathbf{v}\|_{0, \Omega},
\end{aligned}
$$

where $C$ depends only on the shape of $\Omega$ and $\Gamma_{D}$, but not on $\mathrm{d}(\Omega)$.

The last variant of $\mathbf{H}$ (div) regular decomposition of $\mathbf{H}$ (div) dispenses with the assumptions on the topology of the Dirichlet boundary and has better stability properties than the splitting from Theorem 8 (though with less explicit constants).

Theorem 9 (Divergence-Bounded Regular Decomposition of $\mathbf{H}$ (div) (II)) Let $\left(\Omega, \Gamma_{D}\right)$ be as in Sect. 2. Then, for each $\mathbf{v} \in \mathbf{H}_{\Gamma_{D}}(\operatorname{div}, \Omega)$ there exists $\mathbf{z} \in \mathbf{H}_{\Gamma_{D}}^{1}(\Omega)$ and a divergence-free vector field $\mathbf{h} \in \mathbf{H}_{\Gamma_{D}}(\operatorname{div} 0, \Omega)$ depending linearly on $\mathbf{v}$ such that

$$
\begin{aligned}
\mathbf{v} & =\mathbf{z}+\mathbf{h}, \\
\|\mathbf{z}\|_{0, \Omega}+\|\mathbf{h}\|_{0, \Omega} & \leq\|\mathbf{v}\|_{0, \Omega}, \\
\|\nabla \mathbf{z}\|_{0, \Omega}+\frac{1}{\mathrm{~d}(\Omega)}\|\mathbf{z}\|_{0, \Omega} & \leq C\|\operatorname{div} \mathbf{v}\|_{0, \Omega},
\end{aligned}
$$

where $C$ depends only on the shape of $\Omega$ and $\Gamma_{D}$, but not on $\mathrm{d}(\Omega)$.

\section{Discrete Counterparts of the Regular Decompositions}

The discrete setting to which we want to extend the concept of regular decompositions is provided by finite element exterior calculus (FEEC, [2]) which introduces finite element subspaces of $\mathbf{H}$ (curl) and $\mathbf{H}$ (div) as special instances of spaces of discrete differential forms. In this section we confine ourselves to the lowest-order case of piecewise linear finite element functions.

Throughout, we assume that $\left(\Omega, \Gamma_{D}\right)$ is as in Sect. 2, and, additionally, that $\Omega$ is a polyhedron and that $\partial \Gamma_{D}$ consists of straight line segments. All considerations take for granted a shape-regular family of meshes $\left\{\mathcal{T}^{h}\right\}_{h}$ of $\Omega$, consisting of tetrahedral elements, and resolving $\Gamma_{D}$ in the sense that $\Gamma_{D}$ is a union of faces of some of the tetrahedra.

The following finite element spaces will be relevant:

- the space $\mathcal{W}_{h, \Gamma_{D}}^{0}(\Omega)$ of $H_{\Gamma_{D}}^{1}(\Omega)$-conforming piecewise linear Lagrangian finite element functions,

- the space $\mathcal{W}_{h, \Gamma_{D}}^{1}(\Omega)$ of $\mathbf{H}_{\Gamma_{D}}(\mathbf{c u r l}, \Omega)$-conforming lowest order Nédélec elements, also known as edge elements, 
- the space $\mathcal{W}_{h, \Gamma_{D}}^{2}(\Omega)$ of $\mathbf{H}_{\Gamma_{D}}(\operatorname{div}, \Omega)$-conforming lowest order tetrahedral Raviart-Thomas finite elements, aka, face elements,

- the space $\mathcal{W}_{h, \Gamma_{D}}^{0}(\Omega):=\left[\mathcal{W}_{h, \Gamma_{D}}^{0}(\Omega)\right]^{3}$ of piecewise linear globally continuous vector fields vanishing on $\Gamma_{D}$.

Functions in $\mathcal{W}_{h, \Gamma_{D}}^{\ell}(\Omega), \ell=1,2,3$, are so-called Whitney forms, lowest-order discrete differential forms of the first family as introduced in [13] and [2, Sect. 5].

\subsection{Discrete Regular Decompositions for Edge Elements}

Commuting projectors, also known as co-chain projectors, are the linchpin of FEEC theory [2, Sect. 7], and it is not different with our developments. Thus, let

$$
\begin{array}{ll} 
& R_{h, \Gamma_{D}}^{0}: H_{\Gamma_{D}}^{1}(\Omega) \rightarrow \mathcal{W}_{h, \Gamma_{D}}^{0}(\Omega) \\
\text { and } \quad \mathbf{R}_{h, \Gamma_{D}}^{1}: \mathbf{H}_{\Gamma_{D}}(\mathbf{c u r l}, \Omega) \rightarrow \mathcal{W}_{h, \Gamma_{D}}^{1}(\Omega)
\end{array}
$$

denote the continuous, boundary-aware cochain projectors from [17, Sect. 3.2.6], which extend the pioneering work [10] by Falk and Winther. These two linear operators are projectors onto their ranges, they fulfill the commuting property

$$
\nabla\left(R_{h, \Gamma_{D}}^{0} \varphi\right)=\mathbf{R}_{h, \Gamma_{D}}^{1}(\nabla \varphi) \quad \forall \varphi \in H_{\Gamma_{D}}^{1}(\Omega),
$$

and local stability estimates

Theorem 10 ([17, Thm. 1.2]) For each $\mathbf{v}_{h} \in \mathcal{W}_{0, \Gamma_{D}}^{1}(\Omega)$ there exists a continuous and piecewise linear vector field $\mathbf{z}_{h} \in \mathcal{W}_{h, \Gamma_{D}}^{0}(\Omega)$, a continuous and piecewise linear scalar function $p_{h} \in \mathcal{W}_{h, \Gamma_{D}}^{0}(\Omega)$, and a remainder $\widetilde{\mathbf{v}}_{h} \in \mathcal{W}_{0, \Gamma_{D}}^{1}(\Omega)$, all depending linearly on $\mathbf{v}_{h}$, providing the discrete regular decomposition

$$
\mathbf{v}_{h}=\mathbf{R}_{h, \Gamma_{D}}^{1} \mathbf{z}_{h}+\widetilde{\mathbf{v}}_{h}+\nabla p_{h}
$$

and satisfying the stability estimates

$$
\begin{aligned}
\left\|\mathbf{z}_{h}\right\|_{0, \Omega}+\left\|\nabla p_{h}\right\|_{0, \Omega}+\left\|\widetilde{\mathbf{v}}_{h}\right\|_{0, \Omega} & \leq C\left\|\mathbf{v}_{h}\right\|_{0, \Omega}, \\
\left\|\nabla \mathbf{z}_{h}\right\|_{0, \Omega}+\left\|h^{-1} \widetilde{\mathbf{v}}_{h}\right\|_{0, \Omega} & \leq C\left(\left\|\operatorname{curl} \mathbf{v}_{h}\right\|_{0, \Omega}+\frac{1}{\mathrm{~d}(\Omega)}\left\|\mathbf{v}_{h}\right\|_{0, \Omega}\right),
\end{aligned}
$$

where $C$ is a generic constant that depends only on the shape of $\left(\Omega, \Gamma_{D}\right)$, but not on $\mathrm{d}(\Omega)$, and on the shape regularity constant of $\mathcal{T}^{h}(\Omega)$. Above, $h^{-1}$ is the piecewise constant function that is equal to $h_{T}^{-1}$ on every element $T$.

Obviously, this is a discrete counterpart of the regular decomposition of $\mathbf{H}$ (curl) from Theorem 2. The following theorem appears to be new and it corresponds to 
the rotation-bounded regular decomposition of Theorem 5. For the sake of brevity define the discrete nullspace of the curl operator

$$
\mathcal{N}_{h}^{1}:=\left\{\mathbf{v}_{h} \in \mathcal{W}_{h, \Gamma_{D}}^{1}(\Omega): \operatorname{curl} \mathbf{v}_{h}=0\right\}
$$

If $\Omega$ and $\Gamma_{D}$ have simple topology, $\mathcal{X}_{h}=\nabla \mathcal{W}_{h, \Gamma_{D}}^{0}(\Omega)$, but if the first Betti number of $\Omega$ is non-zero, or if $\Gamma_{D}$ has multiple components, then a finite-dimensional cohomology space has to be added [2, Sect. 5.6].

Theorem 11 (Rotation-Bounded Discrete Regular Decomposition for Edge Elements) For each $\mathbf{v}_{h} \in \mathcal{W}_{0, \Gamma_{D}}^{1}(\Omega)$ there exists a continuous and piecewise linear vector field $\mathbf{z}_{h} \in \mathcal{W}_{h, \Gamma_{D}}^{0}(\Omega)$, an curl-free edge element function $\mathbf{h}_{h} \in \mathcal{N}_{h}^{1}$, and a remainder $\widetilde{\mathbf{v}}_{h} \in \mathcal{W}_{0, \Gamma_{D}}^{1}(\Omega)$, all depending linearly on $\mathbf{v}_{h}$, providing the discrete regular decomposition

$$
\mathbf{v}_{h}=\mathbf{R}_{h, \Gamma_{D}}^{1} \mathbf{z}_{h}+\widetilde{\mathbf{v}}_{h}+\mathbf{h}_{h}
$$

and satisfying the stability bounds

$$
\left.\left.\begin{array}{l}
\left\|\mathbf{z}_{h}\right\|_{0, \Omega} \\
\left\|\mathbf{h}_{h}\right\|_{0, \Omega} \\
\left\|\widetilde{\mathbf{v}}_{h}\right\|_{0, \Omega}
\end{array}\right\} \leq C\left\|\mathbf{v}_{h}\right\|_{0, \Omega}, \quad \begin{array}{c}
\left\|\nabla \mathbf{z}_{h}\right\|_{0, \Omega} \\
\left\|h^{-1} \widetilde{\mathbf{v}}_{h}\right\|_{0, \Omega}
\end{array}\right\} \leq C \| \text { curl } \mathbf{v}_{h} \|_{0, \Omega},
$$

where $C$ is a uniform constant that depends only on the shape of $\left(\Omega, \Gamma_{D}\right)$, but not on $\mathrm{d}(\Omega)$, and on the shape regularity constant of $\mathcal{T}^{h}(\Omega)$.

We stress that the statements of Theorems 10 and 11 do not hinge on any assumptions on the topological properties of $\Omega$ and $\Gamma_{D}$.

\subsection{Discrete Regular Decompositions for Face Elements}

For face elements, the construction of a boundary-aware co-chain projection operator

$$
\mathbf{R}_{h, \Gamma_{D}}^{2}: \mathbf{H}_{\Gamma_{D}}(\operatorname{div}, \Omega) \rightarrow \mathcal{W}_{h, \Gamma_{D}}^{2}(\Omega)
$$

that commutes with $\mathbf{R}_{h, \Gamma_{D}}^{1}$ and the curl-operator has not yet been accomplished. Fortunately, in the case $\Gamma_{D}=\emptyset$, this operator is available from [10]. Thus, in the following, we treat only the case $\Gamma_{D}=\emptyset$ and just omit the subscript $\Gamma_{D}$. Then, from [10] we can borrow a linear operator $\mathbf{R}_{h}^{2}: \mathbf{H}(\operatorname{div}, \Omega) \rightarrow \mathcal{W}_{h}^{2}(\Omega)$ such that

$$
\operatorname{curl} \mathbf{R}_{h}^{1} \mathbf{u}=\mathbf{R}_{h}^{2} \operatorname{curl} \mathbf{u} \quad \forall \mathbf{u} \in \mathbf{H}(\operatorname{curl}, \Omega) .
$$

The next result takes Theorem 6 to the discrete setting. 
Theorem 12 (Discrete Regular Decomposition of $\mathcal{W}_{h}^{2}(\Omega)$ ) For each vector field $\mathbf{v}_{h}$ in the lowest-order Raviart-Thomas space $\mathcal{W}_{h}^{2}(\Omega)$, there exists a continuous and piecewise linear vector field $\mathbf{z}_{h} \in \mathcal{W}_{h}^{0}(\Omega)$, a vector field $\mathbf{q}_{h}$ in the lowest-order Nédélec space $\mathcal{W}_{h}^{1}(\Omega)$, and a remainder $\widetilde{\mathbf{v}}_{h} \in \mathcal{W}_{h}^{2}(\Omega)$, all depending linearly on $\mathbf{v}_{h}$, providing the discrete regular decomposition

$$
\mathbf{v}_{h}=\mathbf{R}_{h}^{2} \mathbf{z}_{h}+\widetilde{\mathbf{v}}_{h}+\operatorname{curl} \mathbf{q}_{h},
$$

and the stability estimates

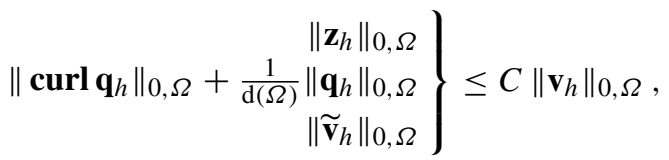

$$
\begin{aligned}
& \left.\begin{array}{r}
\left\|\boldsymbol{\nabla} \mathbf{z}_{h}\right\|_{0, \Omega} \\
\left\|h^{-1} \widetilde{\mathbf{v}}_{h}\right\|_{0, \Omega}
\end{array}\right\} \leq C\left\|\operatorname{div} \mathbf{v}_{h}\right\|_{0, \Omega}+\frac{1}{\mathrm{~d}(\Omega)}\left\|\mathbf{v}_{h}\right\|_{0, \Omega} .
\end{aligned}
$$

The constant $C$ depends only on the shape of $\Omega$, but not on $\mathrm{d}(\Omega)$, and the shaperegularity of $\mathcal{T}^{h}(\Omega)$.

Finally, we present a counterpart to the divergence-bounded regular decomposition of Theorem 9. For convenience we introduce the space of divergence-free face element functions

$$
\mathcal{N}_{h}^{2}:=\left\{\mathbf{q}_{h} \in \mathcal{W}_{h}^{2}(\Omega): \operatorname{div} \mathbf{q}_{h}=0\right\}
$$

Theorem 13 (Divergence-Bounded Discrete Regular Decomposition of $\left.\mathcal{W}_{h}^{2}(\Omega)\right)$ For each vector field $\mathbf{v}_{h}$ in the lowest-order Raviart-Thomas space $\mathcal{W}_{h}^{2}(\Omega)$, there exists a continuous and piecewise linear vector field $\mathbf{z}_{h} \in \mathcal{W}_{h}^{0}(\Omega)$, an element $\mathbf{h}_{h}$ in the discrete divergence-free subspace $\mathcal{N}_{h}^{2}$, and a remainder $\tilde{\mathbf{v}}_{h} \in \mathcal{W}_{h}^{2}(\Omega)$, all depending linearly on $\mathbf{v}_{h}$, providing the discrete regular decomposition

$$
\mathbf{v}_{h}=\mathbf{R}_{h}^{2} \mathbf{z}_{h}+\widetilde{\mathbf{v}}_{h}+\mathbf{h}_{h}
$$

and the stability estimates

$$
\left.\left.\begin{array}{l}
\left\|\mathbf{z}_{h}\right\|_{0, \Omega} \\
\left\|\widetilde{\mathbf{v}}_{h}\right\|_{0, \Omega} \\
\left\|\mathbf{h}_{h}\right\|_{0, \Omega}
\end{array}\right\} \leq C\left\|\mathbf{v}_{h}\right\|_{0, \Omega}, \quad \begin{array}{r}
\left\|\nabla \mathbf{z}_{h}\right\|_{0, \Omega} \\
\left\|h^{-1} \widetilde{\mathbf{v}}_{h}\right\|_{0, \Omega}
\end{array}\right\} \leq C\left\|\operatorname{div} \mathbf{v}_{h}\right\|_{0, \Omega}
$$

The constants $C$ depend only on the shape of $\Omega$, but not on $\mathrm{d}(\Omega)$, and the shape regularity of $\mathcal{T}^{h}(\Omega)$. 
Remark 10 The result of Theorem 13 can be viewed as an improvement of the decompositions in [25] which are elaborated for the case of essential boundary conditions on $\partial \Omega$.

Corollary 2 If the second Betti number of $\Omega$ vanishes, that is, if $\partial \Omega$ is connected, then $\mathbf{h}_{h}$ in Theorem 13 can be chosen as $\mathbf{h}_{h}=\operatorname{curl} \mathbf{q}_{h}$ with $\mathbf{q}_{h} \in \mathcal{W}_{h}^{1}(\Omega)$ such that

$$
\mathbf{v}_{h}=\mathbf{R}_{h}^{2} \mathbf{z}+\widetilde{\mathbf{v}}_{h}+\operatorname{curl} \mathbf{q}_{h},
$$

with the bounds

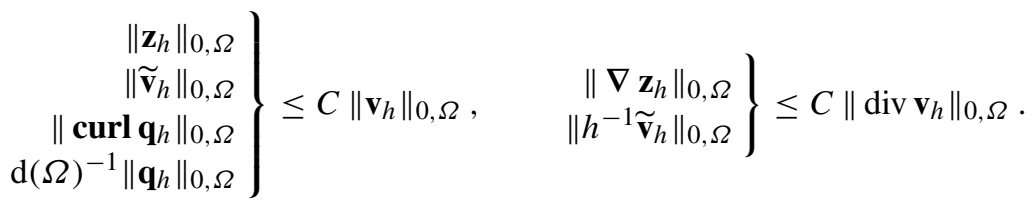

Remark 11 The result of Corollary 2 is an improvement of [19, Lemma 5.2] which assumes a domain $\Omega$ that is smooth enough to allow $H^{2}$-regularity of the Laplace problem (2-regular case, for details see [19, Sect. 3]). This lemma is used in [27] in a domain decomposition framework, where convex subdomains are assumed. With our improved version, this assumption can be weakened considerably.

Acknowledgements The second author would like to thank Dirk Pauly (Essen) for enlightening discussions.

\section{References}

1. Amrouche, C., Bernardi, C., Dauge, M., Girault, V.: Vector potentials in three-dimensional non-smooth domains. Math. Meth. Appl. Sci. 21(9), 823-864 (1998)

2. Arnold, D., Falk, R., Winther, R.: Finite element exterior calculus, homological techniques, and applications. Acta Numer. 15, 1-155 (2006)

3. Bauer, S., Pauly, D., Schomburg, M.: The Maxwell compactness property in bounded weak Lipschitz domains with mixed boundary conditions. SIAM J. Math. Anal. 48(4), 2912-2943 (2016)

4. Birman, M.S., Solomyak, M.Z.: $L^{2}$-theory of the Maxwell operator in arbitrary domains. Usp. Mat. Nauk. 42(6), 61-76 (1987). English Transliterated, Russian Math. Surveys 42(6), 75-96 (1987)

5. Bonnet-Ben Dhia, A., Hazard, C., Lohrengel, S.: A singular field method for the solution of Maxwell's equations in polyhedral domains. SIAM J. Appl. Math. 59(6), 2028-2044 (1999)

6. Buffa, A., Costabel, M., Sheen, D.: On traces for $\mathbf{H}(\mathbf{c u r l}, \Omega)$ in Lipschitz domains. J. Math. Anal. Appl. 276, 845-867 (2002)

7. Costabel, M., Dauge, M., Nicaise, S.: Singularities of Maxwell interface problems. Model. Math. Anal. Numer. 33(2), 627-649 (1999)

8. Creusé, E., Nicaise, S., Tang, Z.: Helmholtz decomposition of vector fields with mixed boundary conditions and an application to a posteriori finite element analysis of the Maxwell system. Math. Methods Appl. Sci. 38(4), 738-750 (2015) 
9. Dautray, R., Lions, J.: Mathematical Analysis and Numerical Methods for Science and Technology. Springer, Berlin (2000). Original French edition published by Masson, S.A., Paris (1984)

10. Falk, R.S., Winther, R.: Local bounded cochain projections. Math. Comp. 83(290), 2631-2656 (2014)

11. Girault, V., Raviart, P.A.: Finite Element Methods for Navier-Stokes Equations. Springer, New York (1986)

12. Gopalakrishnan, J., Qiu, W.: Partial expansion of a Lipschitz domain and some applications. Front. Math. China 7(2), 249-272 (2012)

13. Hiptmair, R.: Canonical construction of finite elements. Math. Comp. 68, 1325-1346 (1999)

14. Hiptmair, R.: Finite elements in computational electromagnetism. Acta Numer. 11, 237-339 (2002)

15. Hiptmair, R.: Boundary element methods for eddy current computation. In: Schanz, M., Steinbach, O. (eds.) Boundary Element Analysis: Mathematical Aspects and Applications. Lecture Notes in Applied and Computational Mechanics, vol. 29, pp. 213-248. Springer, Heidelberg (2007)

16. Hiptmair, R.: Maxwell's equations: Continuous and discrete. In: Bermúdez de Castro, A., Valli, A. (eds.) Computational Electromagnetism. Lecture Notes in Mathematics, vol. 2148. Springer, Cham (2015)

17. Hiptmair, R., Pechstein, C.: Discrete regular decompositions of tetrahedral discrete 1-forms. In: Langer, U., Pauly, D., Repin, S. (eds.) Maxwell's Equations. Radon Series on Computational and Applied Mathematics, vol. 24, pp. 199-258. De Gruyter, Stuttgart (2019)

18. Hiptmair, R., Pechstein, C.: Regular decompositions of vector fields - continuous, discrete, and structure-preserving. Technical Report 2019-18, Seminar for Applied Mathematics, ETH Zürich (2019). https://www.sam.math.ethz.ch/sam_reports/reports_final/reports2019/2019-18. pdf

19. Hiptmair, R., Xu, J.: Nodal auxiliary space preconditioning in $\mathrm{H}$ (curl) and $\mathrm{H}(\mathrm{div})$ spaces. SIAM J. Numer. Anal. 45, 2483-2509 (2007)

20. Hiptmair, R., Zheng, W.: Local multigrid in H(curl). J. Comput. Math. 27(5), 573-603 (2009)

21. Hiptmair, R., Widmer, G., Zou, J.: Auxiliary space preconditioning in $H_{0}$ (curl; $\Omega$ ). Numer. Math. 103, 435-459 (2006)

22. $\mathrm{Hu}, \mathrm{Q} .:$ Convergence of $\mathrm{HX}$ preconditioner for Maxwell's equations with jump coefficients (i): various extensions of the regular Helmholtz decomposition. Technical Report arXiv:1708.05850v2 [math.AP] (2018)

23. Kolev, T.V., Pasciak, J.E., Vassilevski, P.S.: H(curl) auxiliary mesh preconditioning. Numer. Linear Algebr. Appl. 15(5), 455-471 (2008)

24. Kolev, T.V., Vassilevski, P.S.: Parallel auxiliary space AMG for $\mathbf{H}$ (curl) problems. J. Comput. Math. 27(5), 604-623 (2009)

25. Kolev, T.V., Vassilevski, P.S.: Parallel auxiliary space AMG for H(div) problems. SIAM J. Sci. Comput. 34(6), A3079-A3098 (2012)

26. Monk, P.: Finite Element Methods for Maxwell's Equations. Numerical Mathematics and Scientific Computation. Oxford University Press, Oxford (2003)

27. Oh, D., Widlund, O.B., Zampini, S., Dohrmann, C.R.: BDDC algorithms with deluxe scaling and adaptive selection of primal constraints for Raviart-Thomas vector fields. Math. Comp. 87, 659-692 (2018)

28. Pasciak, J.E., Zhao, J.: Overlapping Schwarz methods in $\mathrm{H}$ (curl) on polyhedral domains. Numer. Math. 10(3), 211-234 (2002) 
Open Access This chapter is licensed under the terms of the Creative Commons Attribution 4.0 International License (http://creativecommons.org/licenses/by/4.0/), which permits use, sharing, adaptation, distribution and reproduction in any medium or format, as long as you give appropriate credit to the original author(s) and the source, provide a link to the Creative Commons licence and indicate if changes were made.

The images or other third party material in this chapter are included in the chapter's Creative Commons licence, unless indicated otherwise in a credit line to the material. If material is not included in the chapter's Creative Commons licence and your intended use is not permitted by statutory regulation or exceeds the permitted use, you will need to obtain permission directly from the copyright holder. 